\title{
Cross sections for neutral-current neutrino-nucleus interactions: Applications for ${ }^{12} \mathrm{C}$ and ${ }^{16} \mathrm{O}$
}

\author{
N. Jachowicz, ${ }^{*}$ S. Rombouts, K. Heyde, ${ }^{\dagger}$ and J. Ryckebusch \\ Institute for Theoretical Physics, Vakgroep Subatomaire en Stralingsfysica, Proeftuinstraat 86, B-9000 Gent, Belgium
}

(Received 29 September 1998; revised manuscipt received 4 February 1999)

\begin{abstract}
We study quasielastic neutrino-nucleus scattering on ${ }^{12} \mathrm{C}$ and ${ }^{16} \mathrm{O}$ within a continuum random phase approximation (RPA) model. The RPA equations are solved using a Green's function approach with an effective Skyrme (SkE2) two-body interaction. Besides a comparison with existing calculations, we carry out a detailed study using various methods (Tamm-Dancoff approximation and RPA) and different forces (SkE2 and LandauMigdal). We evaluate cross sections that may be relevant for neutrino nucleosynthesis.

[S0556-2813(99)01606-4]

PACS number(s): 25.30.Pt, 24.10.Eq, 26.30.+k
\end{abstract}

\section{INTRODUCTION}

Neutrinos are extremely well-suited probes to provide detailed information about the structure and properties of the weak interaction, as they are only interacting via the weak forces. Moreover, being intrinsically polarized and coupling to the axial vector as well as to the vector part of the hadronic current, neutrinos are able to reveal other and more precise nuclear structure information than, e.g., electrons do. The most important problem in extracting information from neutrino-nucleus scattering experiments remains the very small interaction cross sections.

These restrictions become rather unimportant when considering astrophysical processes. The amount of neutrinos produced at the end of the lifetime of a massive star during the neutronization of the collapsing core of a star and its subsequent cooling, is as large as $\sim 10^{58}$, representing approximately $99 \%$ of the total released energy $[1,2]$. These supernova neutrinos will play an important role in explosive nucleosynthesis processes, causing a considerable transformation of the material synthesized during the hydrostatic burning phases in the life of the star.

During the last few years, a large number of quasielastic neutrino-nucleus scattering studies have been carried out, including shell model [3], relativistic fermi gas [3,4], and random phase approximation (RPA) calculations using various forces [4-8]. Since both the LSND and the KARMEN Collaborations have been measuring neutrino-nucleus scattering cross sections on ${ }^{12} \mathrm{C}[9,10]$, special attention was paid to the calculation of cross sections for the reactions ${ }^{12} \mathrm{C}\left(\nu_{e}, e^{-}\right) X$ and ${ }^{12} \mathrm{C}\left(\nu_{\mu}, \mu^{-}\right) X$. Despite the theoretical and experimental efforts, no clear agreement between theory and experiment, and between the different theoretical results for the ${ }^{12} \mathrm{C}\left(\nu_{\mu}, \mu^{-}\right){ }^{12} X$ reaction could be reached [8,9,11-13]. Next to these problems, we observe that relatively few neutral-current calculations have been performed $[3,5]$.

In the present work, we examine quasielastic neutralcurrent neutrino-nucleus reactions in an energy region relevant to explosive nucleosynthesis processes, using a continuum random phase approximation (CRPA) formalism (Sec. III). The CRPA equations are solved using a Green's

\footnotetext{
*Corresponding author.

†Present address: CERN CH-1211, Geneva 23, Switzerland.
}

function approach in which the polarization propagator is approximated by an iteration of the first-order contribution [14]. The unperturbed wave functions are generated using either a Woods-Saxon potential or a HF calculation using a Skyrme force. The latter approach makes self-consistent HFRPA calculations possible. Calculations using either a Skyrme or a Landau-Migdal force must give indications about possible differences and sensitivity in methods to the residual two-body interaction used. Finally, we discuss applications for neutral-current reactions on ${ }^{16} \mathrm{O}$ and ${ }^{12} \mathrm{C}$, two nuclei that are very important from an astrophysical point of view. In Sec. VI, we discuss some aspects of neutrino nucleosynthesis and in Sec. VII, the stability of the RPA and Tamm-Dancoff approximation (TDA) methods, using different two-body forces in calculating the neutrino scattering cross section, is discussed.

\section{QUASIELASTIC NEUTRINO SCATTERING}

In the neutral-current neutrino scattering reactions, a neutrino with four-momentum $\left(\epsilon_{i}, \vec{k}_{i}\right)$ is scattered inelastically from a nucleus with initial energy and momentum $E_{i}, \vec{P}_{i}$. The nucleus is supposed to be spherically symmetric and in its ground state, $J_{i}^{\pi_{i}}=0^{+}$. After the reaction, the nucleus is left in an excited state with final parity and angular momentum $J_{f}, \pi_{f}$. The energy transferred from neutrino to nucleus equals $\omega=\epsilon_{i}-\epsilon_{f}=E_{f}-E_{i}, \epsilon_{f}$ and $E_{f}$ being the final neutrino and nuclear energy respectively. The transferred momentum is denoted by $\vec{q}=\vec{k}_{i}-\vec{k}_{f}, \kappa=|\vec{q}|$.

As the interaction between the neutrino and a nucleus is mediated by the weak interaction, the cross section can be derived using Fermi's golden rule. In Born approximation, the outgoing neutrino is described by a plane wave. Using natural units $(\hbar=c=1)$, the differential cross section is then given by

$$
\frac{d^{2} \sigma}{d \Omega d \omega}=(2 \pi)^{4} k_{f} \varepsilon_{f} \sum_{s_{f}, s_{i}} \frac{1}{2 J_{i}+1} \sum_{M_{f}, M_{i}}\left|\left\langle f\left|\hat{H}_{W}\right| i\right\rangle\right|^{2} .
$$

The weak interaction Hamiltonian $\hat{H}_{W}$ has the currentcurrent structure

$$
\hat{H}_{W}=\frac{G}{\sqrt{2}} \int d \vec{x} \hat{j}_{\mu, \text { leptonic }}(\vec{x}) \hat{J}^{\mu, \text { hadronic }}(\vec{x}),
$$


with $\mathrm{G}=1.16639 \times 10^{-11} \mathrm{MeV}^{-2}$ the weak coupling constant. The neutral leptonic current reads

$$
\begin{aligned}
\hat{j}_{\mu}^{0}(x)= & \bar{\Psi}_{\nu_{e}}(x) \gamma_{\mu}\left(1-\gamma_{5}\right) \Psi_{\nu_{e}}(x) \\
& +\bar{\Psi}_{\nu_{\mu}}(x) \gamma_{\mu}\left(1-\gamma_{5}\right) \Psi_{\nu_{\mu}}(x) \\
& +\bar{\Psi}_{\nu_{\tau}}(x) \gamma_{\mu}\left(1-\gamma_{5}\right) \Psi_{\nu_{\tau}}(x) .
\end{aligned}
$$

The weak neutral hadronic current is given by [15]

$$
\begin{aligned}
\hat{J}_{\mu}^{0}= & \hat{J}_{\mu}^{\text {vector }}+\hat{J}_{\mu}^{\text {axial vector }} \\
= & \left(1-2 \sin ^{2} \theta_{W}\right) \hat{J}_{\mu}^{V_{3}}-2 \sin ^{2} \theta_{W} \hat{J}_{\mu}^{S}+\hat{J}_{\mu}^{A_{3}} \\
= & \bar{\Psi}_{N}\left\{G_{E}^{V}\left(q^{2}\right) \gamma_{\mu}+\frac{1}{2 M} G_{M}^{V}\left(q^{2}\right) \sigma_{\mu \nu} q^{\nu}\right. \\
& \left.+G^{A}\left(q^{2}\right) \gamma_{\mu} \gamma_{5}\right\} .
\end{aligned}
$$

Here $\sin ^{2} \theta_{W}=0.2325$, where $\theta_{W}$ is the Weinberg angle. For the isospin operators, the convention $\tau_{3}|p\rangle=+1, \tau_{3}|n\rangle=$ -1 is adopted. $\hat{J}_{\mu}^{V_{3}}$ denotes the $\tau_{3}$ component of the electromagnetic current and $J_{\mu}^{S}$ is its scalar counterpart. The vector form factors can be obtained directly from those in electromagnetic interactions applying the CVC theorem:

$$
\begin{gathered}
G_{E}^{V}=\frac{1}{2}\left(1-2 \sin ^{2} \theta_{W}\right) \tau_{3}-\sin ^{2} \theta_{W}, \\
G_{M}^{V}=\frac{1}{2}\left(1-2 \sin ^{2} \theta_{W}\right)\left(\mu_{p}-\mu_{n}\right) \tau_{3}-\sin ^{2} \theta_{W}\left(\mu_{p}+\mu_{n}\right) .
\end{gathered}
$$

$G^{A}$ is given by

$$
G^{A}=-g_{a} \tau_{3}=-1.262 \tau_{3} .
$$

For low momentum transfers $(\kappa<400 \mathrm{MeV} / c)$, only lowest-order contributions to the hadronic current have to be retained [15]. The differential cross section for neutralcurrent neutrino scattering finally becomes

$$
\left(\frac{d^{2} \sigma_{i \rightarrow f}}{d \Omega d \omega}\right)_{\frac{\nu}{\nu}}=\frac{G^{2} \varepsilon_{f}^{2}}{\pi} \frac{2 \cos ^{2}\left(\frac{\theta}{2}\right)}{2 J_{i}+1}\left[\sum_{J=0}^{\infty} \sigma_{C L}^{J}+\sum_{J=1}^{\infty} \sigma_{T}^{J}\right],
$$

where

$$
\begin{aligned}
\sigma_{C L}^{J}=\left|\left\langle J_{f}|| \hat{\mathcal{M}}_{J}(\kappa)+\frac{\omega}{|\vec{q}|} \hat{\mathcal{L}}_{J}(\kappa)|| J_{i}\right\rangle\right|^{2}, \\
\sigma_{T}^{J}=\left(-\frac{q_{\mu}^{2}}{2|\vec{q}|^{2}}+\tan ^{2}\left(\frac{\theta}{2}\right)\right)\left[\left|\left\langle J_{f} \| \hat{\mathcal{J}}_{J}^{\mathrm{mag}}(\kappa)|| J_{i}\right\rangle\right|^{2}\right. \\
\left.+\left|\left\langle J_{f}|| \hat{\mathcal{J}}_{J}^{\mathrm{el}}(\kappa)|| J_{i}\right\rangle\right|^{2}\right] \mp \tan \left(\frac{\theta}{2}\right) \sqrt{-\frac{q_{\mu}^{2}}{|\vec{q}|^{2}}+\tan ^{2}\left(\frac{\theta}{2}\right)} \\
\times\left[2 \operatorname{Re}\left(\left\langle J_{f}\left\|\hat{\mathcal{J}}_{J}^{\mathrm{mag}}(\kappa)\right\| J_{i}\right\rangle\left\langle J_{f} \| \hat{\mathcal{J}}_{J}^{\mathrm{el}}(\kappa)|| J_{i}\right\rangle^{*}\right)\right] .
\end{aligned}
$$

$\hat{\mathcal{M}}_{J M}$ and $\hat{\mathcal{L}}_{J M}$ denote the Coulomb and longitudinal operator respectively, whereas $\hat{\mathcal{J}}_{J M}^{\mathrm{el}}$ and $\hat{\mathcal{J}}_{J M}^{\mathrm{mag}}$ are the transverse electric and magnetic operators:

$$
\hat{\mathcal{M}}_{J M}(\kappa)=\int d \vec{x}\left[j_{J}(\kappa r) Y_{J}^{M}\left(\Omega_{x}\right)\right] \hat{J}_{o}(\vec{x})
$$

$$
\begin{aligned}
& \hat{\mathcal{L}}_{J M}(\kappa)=\frac{i}{\kappa} \int d \vec{x}\left\{\vec{\nabla}\left[j_{J}(\kappa r) Y_{J}^{M}\left(\Omega_{x}\right)\right]\right\} \hat{\vec{J}}(\vec{x}) \\
& \hat{\mathcal{J}}_{J M}^{\mathrm{el}}(\kappa)=\frac{1}{\kappa} \int d \vec{x}\left\{\vec{\nabla} \times\left[j_{J}(\kappa r) \overrightarrow{\mathcal{Y}}_{J, J}^{M}\left(\Omega_{x}\right)\right]\right\} \hat{\vec{J}}(\vec{x}) \\
& \hat{\mathcal{J}}_{J M}^{\mathrm{mag}}(\kappa)=\int d \vec{x}\left[j_{J}(\kappa r) \overrightarrow{\mathcal{Y}}_{J, J}^{M}\left(\Omega_{x}\right)\right] \cdot \hat{\vec{J}}(\vec{x})
\end{aligned}
$$

\section{THE CONTINUUM RANDOM PHASE APPROXIMATION}

The transition densities necessary to calculate the cross section (8) are determined within a continuum random phase approximation. The unperturbed single-particle wavefunctions are generated by a Hartree-Fock calculation, using a Skyrme potential [16-19]. The CRPA formalism is based on a Green's function approach [14,16,20-22]. The RPA polarization propagator is obtained by the iteration to all orders of the first-order contribution to the particle-hole Green's function

$$
\begin{aligned}
\Pi^{(\mathrm{RPA})}\left(x_{1}, x_{2} ; \omega\right)= & \Pi^{(0)}\left(x_{1}, x_{2} ; \omega\right) \\
& +\frac{1}{\hbar} \int d x \int d x^{\prime} \Pi^{(0)}\left(x_{1}, x ; \omega\right) \\
& \times \widetilde{V}\left(x, x^{\prime}\right) \Pi^{(\mathrm{RPA})}\left(x^{\prime}, x_{2} ; \omega\right),
\end{aligned}
$$

where $\Pi^{(0)}$ denotes the zeroth-order contribution to the polarization propagator. $\widetilde{V}$ is the antisymmetrized form of the residual interaction which in the following will be supposed to be rotationally invariant, allowing us to write $\widetilde{V}\left(x_{1}, x_{2}\right)$ as

$$
\widetilde{V}\left(x_{1}, x_{2}\right)=\sum_{\alpha \beta, J M} U_{\alpha \beta}^{J}\left(r_{1}, r_{2}\right) X_{\alpha}^{J M^{\dagger}}\left(\hat{x}_{1}\right) X_{\beta}^{J M}\left(\hat{x}_{2}\right),
$$

where the $X^{J M}(\hat{x})$ represent spherical tensor operators of rank $J, M$.

A solution to the RPA Bethe-Salpeter equation (10) is given by the set of wave functions implicitly defined by 


$$
\begin{aligned}
\left|\Psi_{C}(E)\right\rangle= & \left|p h^{-1}(E)\right\rangle+\int d x_{1} \int d x_{2} \widetilde{V}\left(x_{1}, x_{2}\right) \sum_{c^{\prime}} \mathcal{P} \int d \epsilon_{p^{\prime}}\left[\frac{\psi_{h^{\prime}}\left(x_{1}\right) \psi_{p^{\prime}}^{\dagger}\left(x_{1}, \epsilon_{p^{\prime}}\right)}{E-\epsilon_{p^{\prime} h^{\prime}}}\left|p^{\prime} h^{\prime-1}\left(\epsilon_{p^{\prime} h^{\prime}}\right)\right\rangle\right. \\
& \left.-\frac{\psi_{h^{\prime}}^{\dagger}\left(x_{1}\right) \psi_{p^{\prime}}\left(x_{1}, \epsilon_{p^{\prime}}\right)}{E+\epsilon_{p^{\prime} h^{\prime}}}\left|h^{\prime} p^{\prime-1}\left(-\epsilon_{p^{\prime} h^{\prime}}\right)\right\rangle\right]\left\langle\Psi_{0}\left|\hat{\psi}^{\dagger}\left(x_{2}\right) \hat{\psi}\left(x_{2}\right)\right| \Psi_{C}(E)\right\rangle,
\end{aligned}
$$

where $\mathcal{P}$ denotes the Cauchy principal value, and the $\left|p h^{-1}(E)\right\rangle$ and $\left|h p^{-1}(-E)\right\rangle$ are the unperturbed particlehole and backward hole-particle solutions of the mean-field problem. The summation index $C$ stands for all quantum numbers defining a reaction channel unambiguously: $C$ $=\left\{n_{h}, l_{h}, j_{h}, m_{h}, \epsilon_{h} ; l_{p}, j_{p}, m_{p}, \tau_{z}\right\}$. The first term in expression (12) corresponds to the Tamm-Dancoff approximation and the second one represents the negative energy RPA contribution. From the energy dependence of the denominators, it is clear that the backward RPA contribution becomes only important for states whose energy eigenvalue deviates substantially from the unperturbed value $\epsilon_{p h}$. This makes RPA a well-suited tool for describing collective excitations in nuclei.

From the wave functions (12) the correctly normalized solutions of the scattering problem can be obtained by taking suitable linear combinations. Defining the $K$ matrix by

$$
\begin{aligned}
K_{C, C^{\prime}}^{J}= & \frac{-1}{2 J+1} \sum_{\alpha, \beta} \int d r_{1} \int d r_{2} U_{\alpha \beta}^{J}\left(r_{1}, r_{2}\right) \\
& \times\left\langle h^{\prime}\left\|X_{\alpha}^{J}\left(x_{1}\right)\right\| p^{\prime}\left(E+\epsilon_{h^{\prime}}\right)\right\rangle_{r_{1}}^{*} \\
& \times\left\langle\Psi_{0}\left\|X_{\beta}^{J}\left(x_{2}\right)\right\| \Psi_{C}(J ; E)\right\rangle_{r_{2}},
\end{aligned}
$$

where the subscript $r$ of the transition densities denotes that all coordinates except the radial one have been integrated, it can be shown that the wave functions constructed by putting

$$
\begin{aligned}
& \left|\Psi_{C}^{+}(J M ; E)\right\rangle \\
& \quad=\sum_{C_{\mathrm{open}}^{\prime}:} \sum_{\epsilon_{p}=\epsilon_{h}+E>0}\left[1+i \pi K^{J}\right]_{C, C^{\prime}}^{-1}\left|\Psi_{C^{\prime}}(J M ; E)\right\rangle
\end{aligned}
$$

contain asymptotically only one incoming wave. They allow us to describe systems where one particle is excited to an unbound state with $\epsilon_{p}>0$, and is able to escape from the nuclear potential. Furthermore they obey the same normalization conditions as the unperturbed $\left|p h^{-1}\right\rangle$ wave functions. From the above, if follows that the wave functions (14) are suited to evaluate the transition densities in the cross section (8).

Defining the unperturbed radial response functions as

$$
\begin{aligned}
& \int d r \int d r^{\prime} R_{\eta \mu ; J M}^{(0)}\left(r, r^{\prime} ; E\right) \\
& \quad=\frac{1}{\hbar} \int d x \int d x^{\prime} X_{\eta J M}(x) \Pi^{(0)}\left(x, x^{\prime} ; \omega\right) X_{\mu J M}^{\dagger}\left(x^{\prime}\right),
\end{aligned}
$$

the RPA transition densities are determined by the set of coupled integral equations

$$
\begin{aligned}
\left\langle\Psi_{0} \|\right. & \left.X_{\eta J} \| \Psi_{C}(J ; E)\right\rangle_{r} \\
= & -\left\langle h\left\|X_{\eta J}\right\| p\left(\epsilon_{p h}\right)\right\rangle_{r} \\
& +\sum_{\mu, \nu} \int d r_{1} \int d r_{2} U_{\mu \nu}^{J}\left(r_{1}, r_{2}\right) \operatorname{Re}\left(R_{\eta \mu ; J}^{(0)}\left(r, r_{1} ; E\right)\right) \\
& \times\left\langle\Psi_{0}\left\|X_{\nu J}\right\| \Psi_{C}(J ; E)\right\rangle_{r_{2}} .
\end{aligned}
$$

Discretizing these equations on a mesh in the radial coordinate, the transition densities for each reaction channel (16) are obtained as the solutions of the matrix equation

$$
\rho_{C}^{\mathrm{RPA}}=-\frac{1}{1-R U} \rho_{C}^{\mathrm{HF}} .
$$

Here $\rho^{\mathrm{RPA}}$ and $\rho^{\mathrm{HF}}$ represent column vectors containing the RPA and the Hartree-Fock transition densities for all included interaction channels $\eta$ and for a number of mesh points in coordinate space. $R$ and $U$ are block matrices containing the unperturbed response function (15) and the radial part of the interaction (11), evaluated at the appropriate channels and $r$ values. The discretization in coordinate space is well under control. It does not demand large numbers of mesh points for the calculated transition densities to become mesh independent, thus keeping the dimension of matrix inversions to be performed sufficiently small. From the form of Eq. (17) it is clear that the wave functions (12) can be considered as the solution to the RPA equivalent of the Lippmann-Schwinger integral scattering equations. In Eq. (17) as well as in Eq. (16), the minus sign arises from the phase convention adopted in the definition of the $K$ matrix (13). This formalism has the interesting feature that treating the RPA equations in coordinate space allows us to deal with

TABLE I. Parameter set for the SkE2-interaction.

\begin{tabular}{lccccccc}
\hline \hline$t_{0}$ & $t_{1}$ & $t_{2}$ & $t_{3}$ & $t_{4}$ & $x_{0}$ & $W_{0}$ & $x_{3}$ \\
\hline-1299.30 & 802.41 & -67.89 & 19558.96 & -15808.79 & 0.270 & 120 & 0.43 \\
$\mathrm{MeV} \mathrm{fm}^{3}$ & $\mathrm{MeV} \mathrm{fm}^{5}$ & $\mathrm{MeV} \mathrm{fm}^{5}$ & $\mathrm{MeV} \mathrm{fm}^{6}$ & $\mathrm{MeV} \mathrm{fm}^{8}$ & & $\mathrm{MeV} \mathrm{fm}^{5}$ & \\
\hline \hline
\end{tabular}




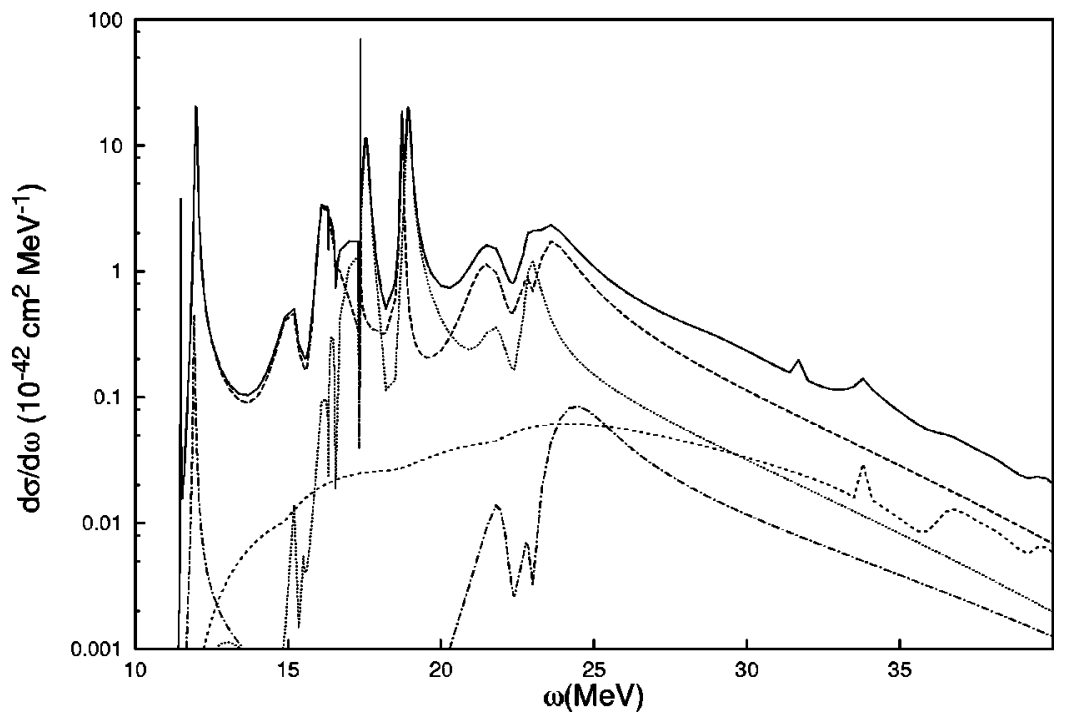

FIG. 1. Cross section for the reaction ${ }^{16} \mathrm{O}$ $+\nu_{50 \mathrm{MeV}} \rightarrow{ }^{16} \mathrm{O}^{*}+\nu^{\prime}$ (full line) and its dominant multipole contributions. $J^{\pi}=1^{-}$(dashed line), $J^{\pi}=1^{+}$(small dashes below), $J^{\pi}=2^{-}$ (dotted line), and $J^{\pi}=0^{-}$(dashed-dotted). The total cross section includes multipoles up to $J$ $=4$.

the energy continuum in an exact way, without cutoff or discretization of the excitation energies [14].

\section{THE SkE2 INTERACTION}

The Hartree-Fock and RPA calculations were performed with an extended Skyrme force. The parameter values used to obtain the presented results are those of the SkE2 parametrization [16-19]. This parameter set was designed to yield a realistic description of nuclear structure properties in both the particle-particle (pairing properties) and in the particle- hole channels and this over the whole mass table. This is done by replacing part of the three-particle contribution to the original Skyrme versions by a momentum dependent two-particle term. The extra free parameter thus obtained is used to guarantee correct two-body characteristics in nuclei containing few valence nucleons outside of the closed shells. Furthermore, the SkE2 parameter set allows a good reproduction of the experimental single-particle energies [16-19].

In coordinate space, the antisymmetrized residual interaction takes the form

$$
\begin{aligned}
V\left(\vec{r}_{1}, \vec{r}_{2}\right)= & t_{0}\left(1+x_{0} \hat{P}_{\sigma}\right) \delta\left(\vec{r}_{1}-\vec{r}_{2}\right)-\frac{1}{8} t_{1}\left[\left(\overleftarrow{\nabla}_{1}-\overleftarrow{\nabla}_{2}\right)^{2} \delta\left(\vec{r}_{1}-\vec{r}_{2}\right)+\delta\left(\vec{r}_{1}-\vec{r}_{2}\right)\left(\vec{\nabla}_{1}-\vec{\nabla}_{2}\right)^{2}\right]+\frac{1}{4} t_{2}\left(\overleftarrow{\nabla}_{1}-\overleftarrow{\nabla}_{2}\right) \delta\left(\vec{r}_{1}-\vec{r}_{2}\right) \\
& \times\left(\vec{\nabla}_{1}-\vec{\nabla}_{2}\right)+\frac{e^{2}}{\left|\vec{r}_{1}-\vec{r}_{2}\right|}+i W_{0}\left(\vec{\sigma}_{1}+\vec{\sigma}_{2}\right)\left(\overleftarrow{\nabla}_{1}-\overleftarrow{\nabla}_{2}\right) \times \delta\left(\vec{r}_{1}-\vec{r}_{2}\right)\left(\vec{\nabla}_{1}-\vec{\nabla}_{2}\right)+\frac{1}{6} t_{3}\left(1-x_{3}\right)\left(1+\hat{P}_{\sigma}\right) \\
& \times \rho\left(\frac{\vec{r}_{1}+\vec{r}_{2}}{2}\right) \delta\left(\vec{r}_{1}-\vec{r}_{2}\right)+x_{3} t_{3} \delta\left(\vec{r}_{1}-\vec{r}_{2}\right) \delta\left(\vec{r}_{1}-\vec{r}_{3}\right)-\frac{1}{24} t_{4}\left\{\left[\left(\overleftarrow{\nabla}_{1}-\overleftarrow{\nabla}_{2}\right)^{2}+\left(\overleftarrow{\nabla}_{2}-\overleftarrow{\nabla}_{3}\right)^{2}+\left(\overleftarrow{\nabla}_{3}-\overleftarrow{\nabla}_{1}\right)^{2}\right]\right. \\
& \left.\times \delta\left(\vec{r}_{1}-\vec{r}_{2}\right) \delta\left(\vec{r}_{1}-\vec{r}_{3}\right)+\delta\left(\vec{r}_{1}-\vec{r}_{2}\right) \delta\left(\vec{r}_{1}-\vec{r}_{3}\right)\left[\left(\vec{\nabla}_{1}-\vec{\nabla}_{2}\right)^{2}+\left(\vec{\nabla}_{2}-\vec{\nabla}_{3}\right)^{2}+\left(\vec{\nabla}_{3}-\vec{\nabla}_{1}\right)^{2}\right]\right\}
\end{aligned}
$$

with $P_{\sigma}$ the spin exchange operator. Table I illustrates the parameter values for the SkE2 set. As the same interaction with the same parameter values is adopted for the calculation of the unperturbed as well as the RPA wave functions, the formalism is self-consistent with respect to the residual interaction used.

\section{APPLICATIONS TO ${ }^{16} \mathrm{O}$ AND ${ }^{12} \mathrm{C}$}

\section{A. The nucleus ${ }^{16} \mathrm{O}$}

As one of the major products of the thermonuclear burning processes in massive stars, ${ }^{16} \mathrm{O}$ plays an important role in supernova nucleosynthesis [1,23]. Moreover, having closed proton and neutron shells, the lack of major nuclear structure difficulties, makes it a good test for the reliability of the formalism. Therefore, the study of neutrino-nucleus interactions with the CRPA formalism was started with crosssection calculations for the neutral-current reaction

$$
{ }^{16} \mathrm{O}+\nu \rightarrow{ }^{16} \mathrm{O}^{*}+\nu^{\prime}
$$

In all of the following results, calculations were performed with an incoming neutrino energy $\epsilon_{i}=50 \mathrm{MeV}$. Multipoles up to $J=4$ were taken into account. Contributions of higher-order multipole excitations were found to be negligibly small. The differential neutrino scattering cross sections are of the order of $10^{-42} \mathrm{~cm}^{2}$ per MeV. In Fig. 1, we show the total cross section and some important multi- 


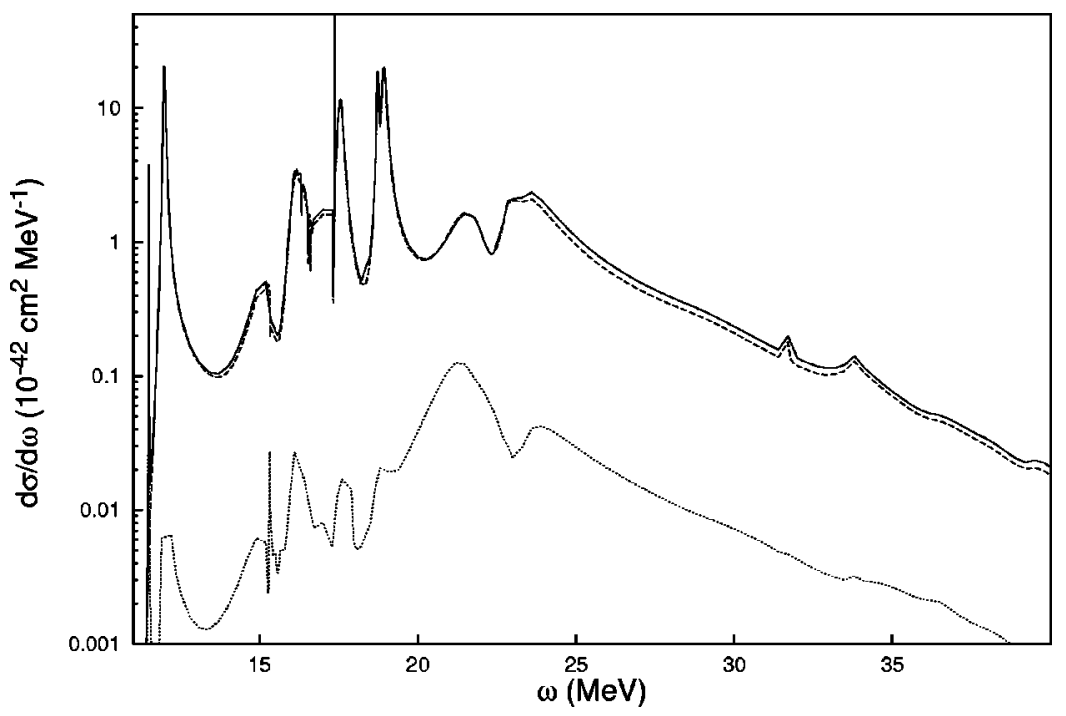

FIG. 2. Comparison between the vector (dotted) and the axial vector (dashed line) contribution to the reaction ${ }^{16} \mathrm{O}\left(\nu, \nu^{\prime}\right){ }^{16} \mathrm{O}^{*}$.

pole contributions. At excitation energies between 20 and 25 $\mathrm{MeV}$, the broad resonance structure of the giant dipole resonance shows up. At energies below $20 \mathrm{MeV}$, smaller peaks, related to excitations with a stronger single-particle character, are present. In RPA results, resonances are pushed to somewhat higher energies than those in mean-field calculations due to the repulsive character of the residual interaction in the isovector channels. For excitation energies above $\sim 30 \mathrm{MeV}$, the cross section decreases almost purely exponentially, according to the energy dependence of Eq. (8). The $J=1$ excitations are clearly prominent. $J=0$ excitations are suppressed due to the fact that only Coulomb and longitudinal terms contribute to these channels. But still, some clear $0^{-}$resonances show up in the differential cross sections.

In Fig. 2, we make a comparison between the contribution of the vector and the axial vector part of the hadronic current to the total cross section. The axial vector current is clearly more sensitive to the weak neutrino probes. The vector contribution is suppressed by almost two orders of magnitude. The splitting of the cross section into a vector and axial vector part excludes the interference contribution. This explains the discrepancy between the sum of both curves in Fig. 2 and the total cross section. Due to the fact that the axial vector current is completely isovector, isovector exci- tations will dominate isoscalar ones. Isoscalar excitations are even further suppressed: not only is the axial vector current not contributing to isoscalar transitions, but due to the $\sin ^{2} \theta_{W}$ factor, the isoscalar form factors are considerably smaller than the vector ones as well.

In Fig. 3 we then compare the contribution of the different operators to the cross section. Transverse transitions are clearly prominent. The difference between the sum of transverse and CL terms and the total cross section is again due to the transverse interference term. According to Eq. (8) it is this interference contribution that is responsible for the difference in the nuclear response to neutrino and antineutrino perturbations. The sign of the interference term determines which cross section will be dominant. Generally neutrino cross sections are slightly larger than antineutrino cross sections. Only at around $23 \mathrm{MeV}$ does the interference term change sign and antineutrino excitations become more important.

Our self-consistent calculations substantiate the results of Kolbe et al. $[5,6]$ on neutral-current reactions. Contrary to the present work, there the CRPA equations are solved using the techniques proposed by Ref. [24]. The standard RPA equations are extended to the continuum by the introduction of an integration over the excitation energies. The resulting

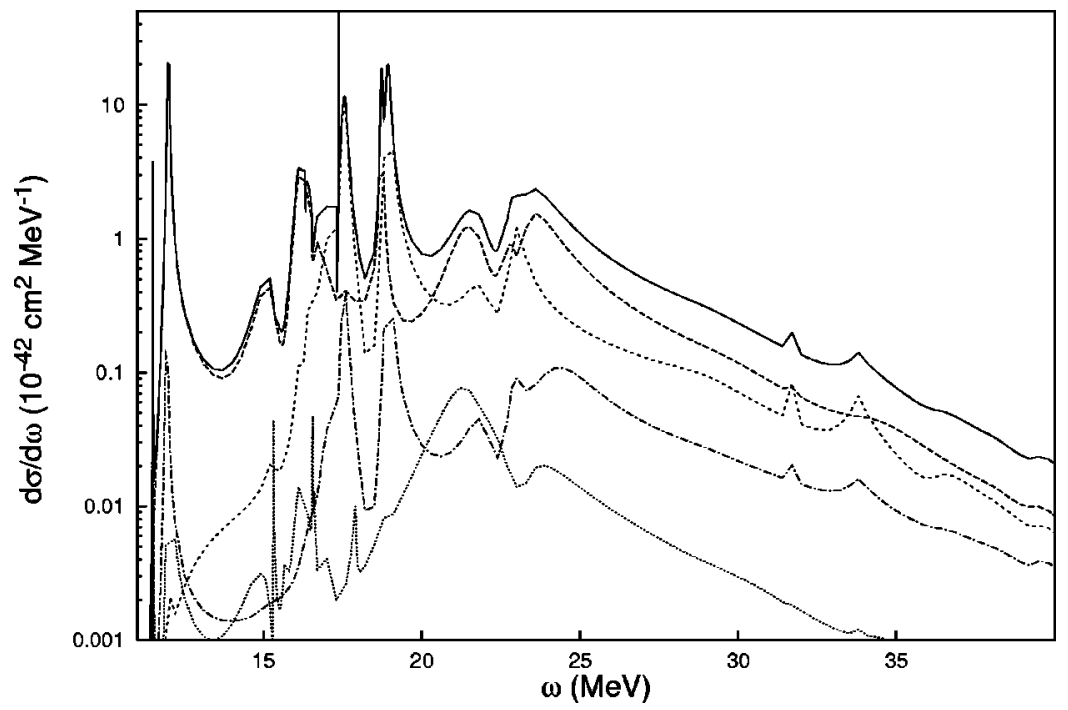

FIG. 3. Coulomb (dotted line), longitudinal (dashed-dotted), transverse electric (short dashed), and transverse magnetic (dashed) contributions to the reaction ${ }^{16} \mathrm{O}\left(\nu, \nu^{\prime}\right){ }^{16} \mathrm{O}^{*}$. The full line gives the total differential cross section. 


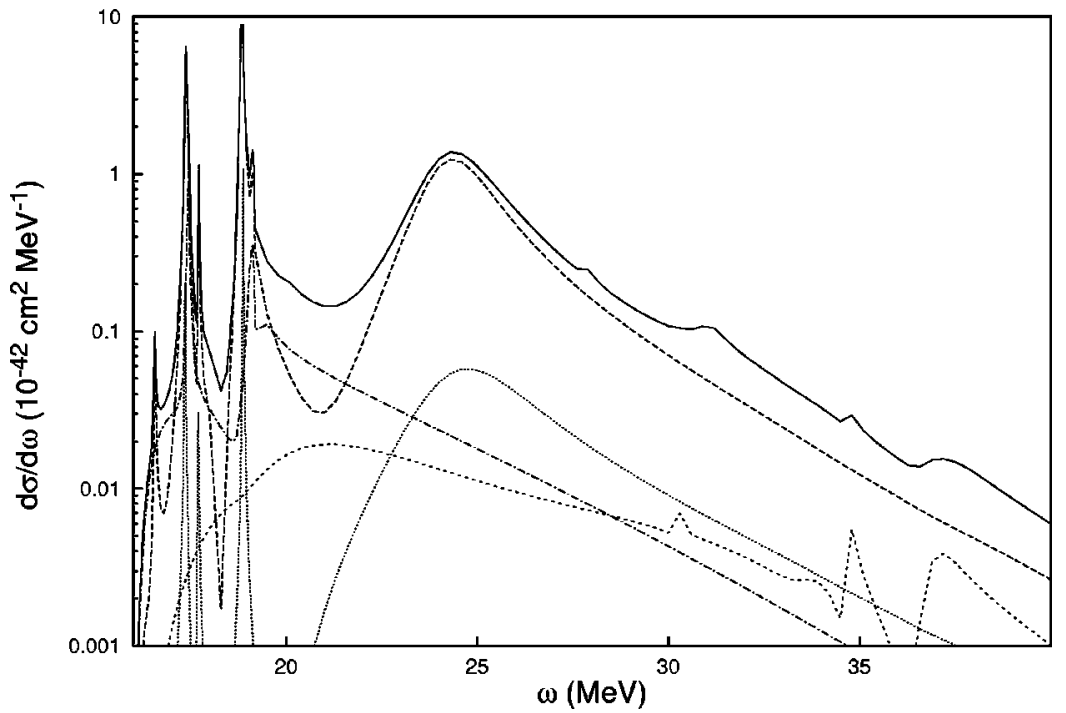

FIG. 4. Cross section for the reaction ${ }^{12} \mathrm{C}$ $+\nu_{50 \mathrm{MeV}} \rightarrow{ }^{12} \mathrm{C}^{*}+\nu^{\prime}$ (full line) and its dominant multipole contributions. $J^{\pi}=1^{-}$(dashed line), $J^{\pi}=1^{+}$(small dashes below), $J^{\pi}=0^{-}$ (dotted line), and $J^{\pi}=2^{-}$(dashed-dotted). The total cross section includes multipoles up to $J$ $=4$.

integrodifferential equations are then solved by an expansion in Weinberg states, already having the correct asymptotic behavior and thus making a fast convergence possible. As residual interaction an effective force derived from the Bonn meson exchange potential is used. The single-particle wave functions are generated using a Woods-Saxon potential. A comparison of results obtained with the different methods makes clear that total cross sections as well as the cross section for transitions to specific $J^{\pi}$ final states show remarkable agreement in overall strength and in the position of the resonances. Moreover, also the more detailed conclusions concerning relative strength of vector and axial vector, neutrino and antineutrino, isovector and isoscalar, and transverse and Coulomb-longitudinal contributions are in excellent agreement.

\section{B. The nucleus ${ }^{12} \mathrm{C}$}

As ${ }^{12} \mathrm{C}$ is known to be deformed in its ground state, whereas the Hartree-Fock calculation assumes the nucleus to have a spherical ground state, the single-particle wave functions for ${ }^{12} \mathrm{C}$ were determined in a different way: the unperturbed wave functions were generated with a Woods-Saxon potential, yielding a more reliable splitting between the single-particle energy levels. The Woods-Saxon parameters were taken from Ref. [25]. Thereby, the full self-consistency is spoiled but it is clearly an approach that allows us to take into account some effects of the deformed ground state, albeit in a semiphenomenological way.

Cross sections for neutral-current neutrino scattering on ${ }^{12} \mathrm{C}$ show mainly the same behavior as those for the reaction ${ }^{16} O\left(\nu, \nu^{\prime}\right){ }^{16} O^{*}$. In Fig. 4 we show the differential cross section and the principal multipole contributions $J^{\pi}$ $=1^{+}, 1^{-}$, and $2^{-}$. Again axial vector and isovector excitations are prominent. Coulomb and longitudinal contributions are suppressed. Neutrino cross sections dominate antineutrino excitations. As was the case for ${ }^{16} \mathrm{O}$, our results are in good agreement with those of Kolbe et al. [5,6].

\section{NEUTRINO NUCLEOSYNTHESIS}

As neutrinos only participate in weak interaction processes and cross sections for scattering reactions involving neutrinos are very small, the importance of neutrinos to astrophysical processes has long been underestimated. However, models describing the explosion mechanism of type II supernovae provide an important role for neutrinos in these

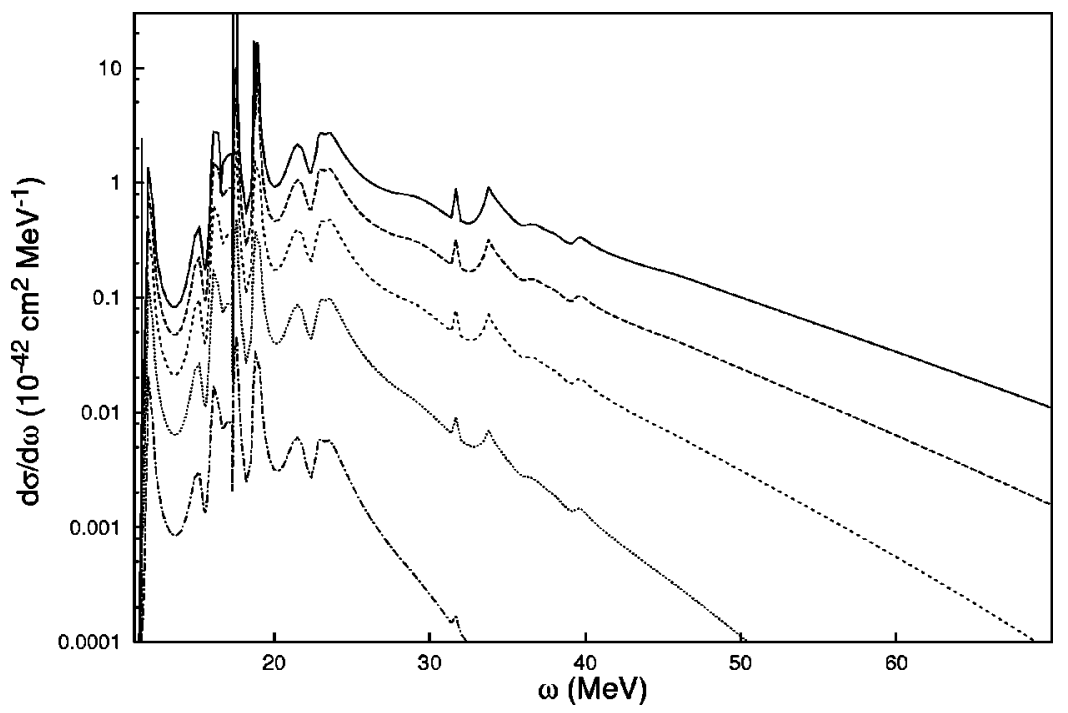

FIG. 5. Differential cross section for the reaction ${ }^{16} \mathrm{O}+\nu_{F D} \rightarrow{ }^{16} \mathrm{O}^{*}+\nu^{\prime}$, averaged over neutrinos and antineutrinos and over a FermiDirac distribution with temperature $T . T$ $=12 \mathrm{MeV}$ (full line), $T=10 \mathrm{MeV}$ (dashed), $T$ $=8 \mathrm{MeV}$ (short dashed), $T=6 \mathrm{MeV}$ (dotted), and $T=4 \mathrm{MeV}$ (dashed-dotted). 
TABLE II. Cross section per nucleon for the reaction ${ }^{16} \mathrm{O}$ $+\nu_{F D} \rightarrow{ }^{16} \mathrm{O}^{*}+\nu^{\prime}$, averaged over neutrinos and antineutrinos and over a Fermi-Dirac distribution with temperature $T$.

\begin{tabular}{|c|c|c|c|c|c|c|c|}
\hline \multicolumn{3}{|c|}{$T(\mathrm{MeV})$} & 4 & 6 & 8 & 10 & 12 \\
\hline & & MF & 0.0033 & 0.045 & 0.22 & 0.71 & 1.7 \\
\hline & & CRPA & 0.0056 & 0.070 & 0.32 & 1.0 & 2.2 \\
\hline
\end{tabular}

processes [1,2]. The cooling of a newly formed neutron star by the production and subsequent emission of neutrino pairs causes a flux of $\sim 10^{58}$ neutrinos, representing an energy of $10^{53} \mathrm{erg}$. Although the neutrinos are only weakly interacting, this enormous amount of particles and energy traveling through the different layers of the star is able to cause a considerable transformation of the elements synthesized during the thermonuclear burning processes in the life of the star.

The energy distribution of the supernova neutrinos is described by a Fermi-Dirac spectrum [1]

$$
n_{\nu}(E, T)=\frac{N}{T^{3}} \frac{E^{2}}{1+e^{E / T}},
$$

where the normalization factor $N$ equals 0.533 . The temperature of the spectra amounts to approximately $3.5 \mathrm{MeV}$ for electron neutrinos and $5 \mathrm{MeV}$ for electron antineutrinos [26]. As typical supernova energies are not high enough to produce heavy leptons, heavy flavor neutrinos do not participate in charged current reactions. They escape from regions closer to the center of the star with temperatures of $8-10$ $\mathrm{MeV}$. As the cross sections are roughly proportional to the square of the incoming neutrino energy, the higher temperature $\mu$ and $\tau$ neutrinos and neutral-current reactions will dominate nucleosynthesis processes.

In Fig. 5 we show the results for calculations where the ${ }^{16} \mathrm{O}$ cross sections have been folded with a Fermi-Dirac spectrum with temperatures between 4 and $12 \mathrm{MeV}$. Table II summarizes the total cross sections for the Hartree-Fock and the CRPA calculation. The results obtained in the present
TABLE III. Cross section per nucleon for the reaction ${ }^{12} \mathrm{C}$ $+\nu_{F D} \rightarrow{ }^{12} \mathrm{C}^{*}+\nu^{\prime}$, averaged over neutrinos and antineutrinos and over a Fermi-Dirac distribution with temperature $T$.

\begin{tabular}{|c|c|c|c|c|c|c|}
\hline$T(\mathrm{MeV})$ & & 4 & 6 & 8 & 10 & 12 \\
\hline & MF & 0.0018 & 0.024 & 0.12 & 0.41 & 1.0 \\
\hline & CRPA & 0.0016 & 0.026 & 0.14 & 0.45 & 1.1 \\
\hline
\end{tabular}

study are in good agreement with those of Ref. [5] and [1] and thus substantiate the reliability (cross section magnitude and overall shape) of CRPA calculations determining neutrino-nucleus scattering processes.

The sensitivity of the cross sections to the temperature is due to the strong energy dependence of the nuclear response in the considered energy region. The temperatures studied correspond to average neutrino energies between 12 and 38 $\mathrm{MeV}$. The large differences are then easily explained by noting that the continuum in ${ }^{16} \mathrm{O}$ only opens at $\sim 11 \mathrm{MeV}$. Next to a bare energy effect, the number of particles with energies above the ${ }^{16} \mathrm{O}$ particle threshold increases considerably with the temperature of the neutrino distribution, enhancing the cross sections accordingly. As a consequence, it is mainly the neutrinos in the high-energy tail of the spectrum that are responsible for the excitation of nuclei. It is therefore important to have a good description of this part of the energy spectrum for supernova neutrinos. Monte Carlo simulations of neutrino transport in supernovae indicate that in this energy region, the spectrum is somewhat depleted compared to the distribution (20). The tail of the Fermi-Dirac distribution can be adjusted to a more accurate reproduction of the actual supernova spectrum by bringing in a chemical potential $[27,28]$. The new neutrino energy spectrum then reads

$$
n_{\nu}(E, T, \alpha)=\frac{N_{\alpha}}{T^{3}} \frac{E^{2}}{1+e^{(E / T+\alpha)}},
$$

where $\alpha$ is the parameter associated with the nonzero chemical potential and $N_{\alpha}$ is a normalization factor depending on

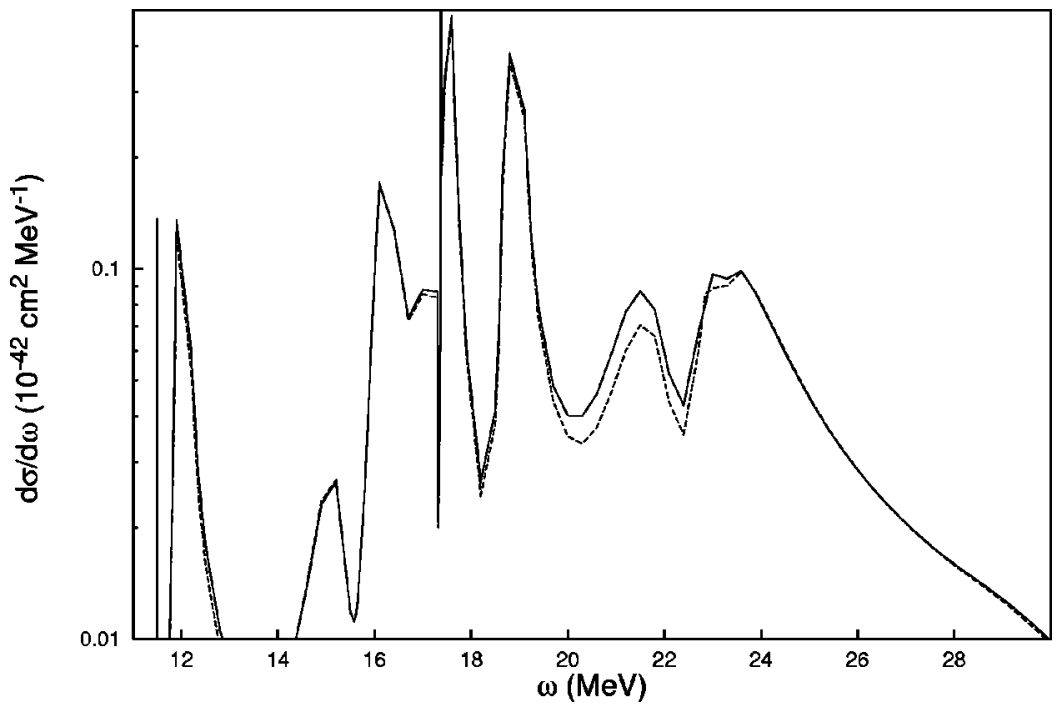

FIG. 6. Differential cross section for the reaction ${ }^{16} \mathrm{O}+\nu_{F D} \rightarrow{ }^{16} \mathrm{O}^{*}+\nu^{\prime}$, averaged over neutrinos and antineutrinos and over a FermiDirac distribution with temperature $T=6 \mathrm{MeV}$, $\alpha=0$ (full line) and $\alpha=5$ (dashed). 


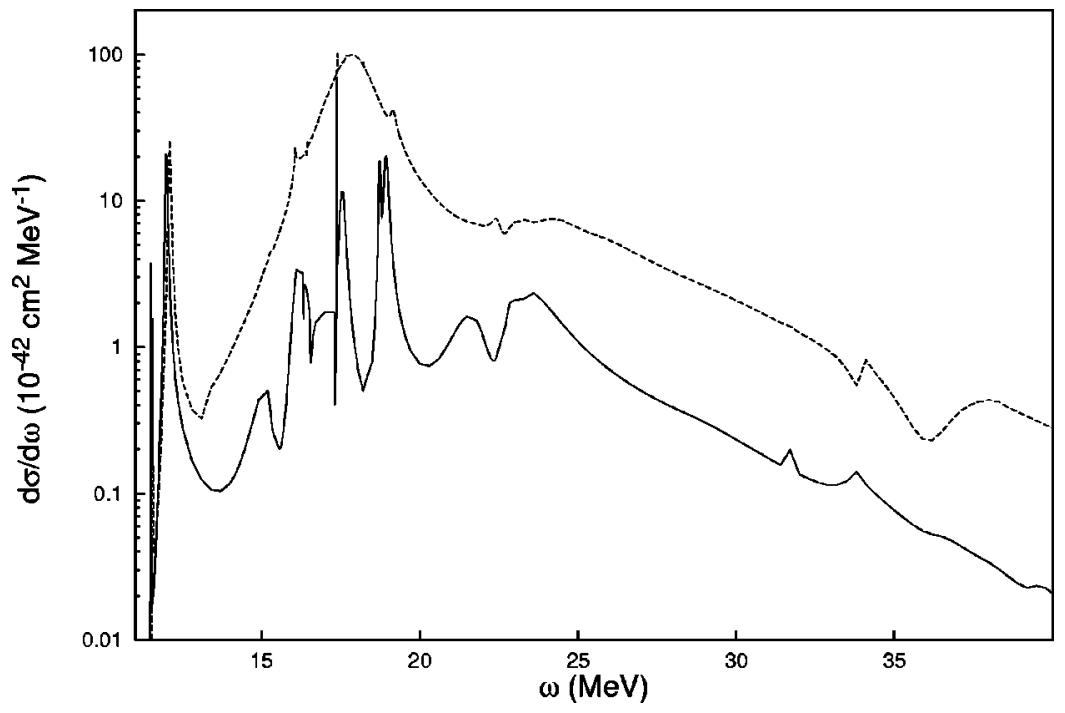

FIG. 7. Comparison of RPA (full line) to TDA results (dashed) for the reaction ${ }^{16} \mathrm{O}\left(\nu, \nu^{\prime}\right){ }^{16} \mathrm{O}^{*}$. The discrepancy is most pronounced in the $J^{\pi}=1^{+}, 2^{-}$, and $0^{+}$channels. The incoming neutrino energy is $50 \mathrm{MeV}$. All multipoles up to $J=4$ are taken into account.

$\alpha$. In Fig. 6 we show how the introduction of a nonzero $\alpha$ parameter affects the differential cross sections. The influence of such an adjustment on the total cross sections is relatively small. Only in the giant dipole resonance region the adjustment of the neutrino spectrum causes slight changes in the results. Further investigations showed that differences caused by the variation of the parameter $\alpha$ in the range $\alpha=3$ to $\alpha=6$ have a negligible influence on the results.

In Table III we show a similar picture for neutrino nucleosynthesis reactions on ${ }^{12} \mathrm{C}$. Here, similar conclusions as in the case of ${ }^{16} \mathrm{O}$ can be drawn in comparison with other calculations.

\section{ON THE RELIABILITY OF THE TDA AND RPA CALCULATIONS}

Starting from the CRPA calculations, as discussed in Sec. $\mathrm{V}$ and VI, one can easily evaluate TDA results by switching off the backward-going term in the wave functions [see expression (12)]. Due to the energy factors appearing in the denominators of Eq. (12), the latter terms are not expected to have a major influence on the resulting transition densities. Nevertheless, the comparison of the TDA and RPA results in
Fig. 7 shows some important differences. The contribution of $1^{+}, 2^{-}$, and $0^{+}$excitations are unproportionally large in TDA, compared to those in RPA.

Moreover, the TDA solutions are unstable against small changes in the residual interaction. This can easily be illustrated by using different two-body forces. In Fig. 8 we compare the RPA and TDA results using the SkE2-Skyrme and Landau-Migdal forces. The Landau-Migdal parameters were taken from Ref. [29]. Apart from slight differences in the relative weight of the resonances, the RPA cross sections obtained using different residual interactions are in good agreement. The outcome of the TDA calculations, however, is extremely sensitive to the force used, as is illustrated in Fig. 8 .

The anomalous behavior of the TDA solution may well be caused by the intrinsic asymmetry underlying the TDA equations [30]. The TDA accepts the shell-model closed-shell configuration as the ground state, whereas excited states are described by a coherent superposition of particle-hole excitations. The random phase approximation considers particlehole and negative energy hole-particle configurations out of a correlated ground state. Whereas in the RPA formalism the ground state is treated on an equal basis with all excited

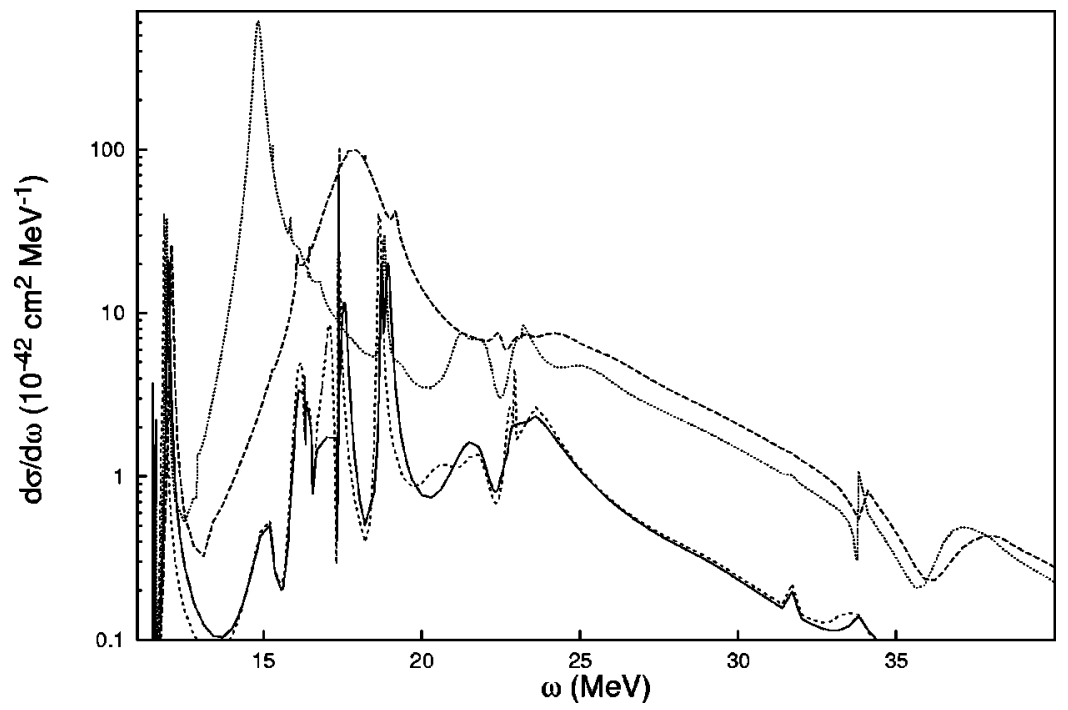

FIG. 8. Comparison of the dependence of RPA and TDA results on the residual interaction used. Whereas RPA calculations are stable against changes in the interaction, TDA cross sections are not. RPA-SkE2 (full line), RPA-LM (short dashed), TDA-SkE2 (dashed), TDA-LM (dotted). 


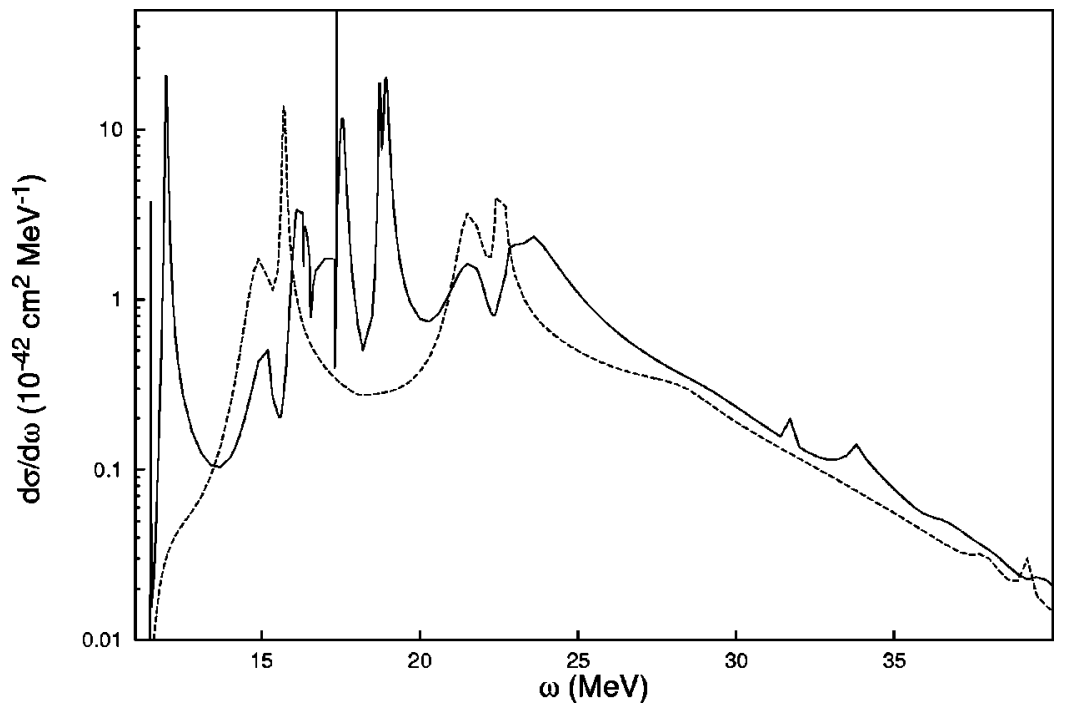

FIG. 9. Comparison of RPA (full line) to Hartree-Fock (dashed) results. The incoming neutrino energy is $50 \mathrm{MeV}$. All multipoles up to $J$ $=4$ are taken into account. The large difference between the random phase and the mean-field response at low excitation energies indicates that RPA calculations in this energy region may not give as accurate a description as one could expect. For excitation energies above $\sim 27 \mathrm{MeV}$, RPA calculations provide a reasonable correction to the mean-field ones. states, the lack of backward going terms in the TDA equations implies that the TDA vacuum is a static reference state.

Furthermore it should be stressed that even the RPA results have to be considered with extreme care, especially for excitation energies below $\sim 25 \mathrm{MeV}$. The large contributions introduced by the RPA correlations compared to the Hartree-Fock results, as is illustrated in Fig. 9, make it difficult to see the RPA as a correction that can be handled within perturbation theory. These differences are indications that more complex nuclear configurations should be incorporated in order to give a more reliable description of both the resonance energies and widths in the giant resonance region. A related problem is the sensitivity of the results to the choice of single-particle energies and wave functions, which makes it hard to obtain unambiguous cross section results.

\section{CONCLUSION}

In the present paper, we have carried out RPA studies of neutrino-nucleus scattering reactions and corresponding nucleosynthesis processes. The RPA equations have been studied using a Green's function approach with an effective Skyrme force (SkE2) and a Landau-Migdal force as residual two-body interactions. The SkE2-force already showed its strength in evaluating various electromagnetic processes. We now have extended applications into the weak interaction sector.

Besides performing detailed calculations for ${ }^{16} \mathrm{O}$ and ${ }^{12} \mathrm{C}$, we have carefully studied the consequences of using TDA instead of RPA, and the effects of using different residual interactions in order to obtain conclusions on the reliability of present-day RPA calculations.

A first important conclusion is that our results substantiate earlier ones, obtained by Kolbe et al. [5,6] using a different technique to solve the RPA equations. Moreover, the RPA results do not seem to be very sensitive to the particular choice of the residual two-body force. We also noted that the TDA results differ substantially from RPA results and thus much caution has to be taken when using the former method. As expected there also appears a clear sensitivity to the use of the particular unperturbed single-particle energy spectrum used as input. This can cause shifts in the resonance structure. These latter conclusions also point towards the need to incorporate more complex configurations in order to produce both the correct excitation energies and widths of the various resonances.

In ${ }^{16} \mathrm{O}$ as well as in ${ }^{12} \mathrm{C}$ the dominant multipole transitions are $J^{\pi}=1^{-}, 1^{+}, 2^{-}$. The $J=0$ exitations are suppressed. Due to the dominance of the axial vector contribution, isovector excitations are clearly prominent. In both nuclei, neutrino cross sections are slightly larger than cross sections for excitations induced by antineutrinos.

Both nuclei studied play an important role in explosive nucleosynthesis. We made an estimate of the importance of the reactions studied to neutrino nucleosynthesis by folding the neutrino-nucleus scattering cross sections with a FermiDirac energy spectrum. The resulting cross sections depend sensitively on the temperature of the neutrino spectrum. The influence of the introduction of a chemical potential is rather small.

\section{ACKNOWLEDGMENTS}

One of the authors (N.J.) thanks the University Research Board (BOZF), S.R., K.H., and J.R. are grateful to the Fund for Scientific Research (FWO) Flanders for financial support. Moreover, K.H. thanks CERN for financial support in the later stages of this work. The authors are grateful to M. Arnould, S. Goriely, E. Kolbe, K. Langanke, and F. Thieleman for many fruitful discussions.
[1] S.E. Woosley, D.H. Hartmann, R.D. Hofman, and W.C. Haxton, Astrophys. J. 356, 272 (1990).

[2] W.C. Haxton, Nucl. Phys. A553, 397c (1993).

[3] W.M. Alberico, M.B. Barbaro, S.M. Bilenky, J.A. Caballero,
C. Giunti, C. Maieron, E. Moya de Guerra, and J.M. Udías, Nucl. Phys. A623, 471 (1997).

[4] H. Kim, J. Piekarewicz, and C.J. Horowitz, Phys. Rev. C 51, 2739 (1994). 
[5] E. Kolbe, K. Langanke, S. Krewald, and F.K. Thielemann, Nucl. Phys. A540, 599 (1992).

[6] E. Kolbe Ph.D. thesis, Westfälischen Wilhelms-Universität Münster, 1992.

[7] N. Auerbach, N. Van Giai, and O.K. Vorov, Phys. Rev. C 56, 2368 (1997).

[8] S.K. Singh, Nimai C. Mukhopadhyay, and E. Oset, Phys. Rev. C 57, 2687 (1998).

[9] C. Athanassopoulos et al., Phys. Rev. C 56, 2806 (1997).

[10] B. Zeitnitz, Prog. Part. Nucl. Phys. 32, 351 (1994).

[11] E. Kolbe, K. Langanke, F.K. Thielemann, and P. Vogel, Phys. Rev. C 52, 3437 (1995).

[12] E. Kolbe, Phys. Rev. C 54, 1741 (1996).

[13] E. Kolbe, K. Langanke, and P. Vogel, Nucl. Phys. A613, 382 (1997).

[14] J. Ryckebusch, M. Waroquier, K. Heyde, J. Moreau, and D. Ryckbosch, Nucl. Phys. A476, 273 (1988).

[15] J.D. Walecka, Semileptonic Weak Interactions in Nuclei in Muon Physics II - Weak Interactions, edited by V.W. Hughes and C.S. Wu (Academic, New York, 1975).

[16] J. Ryckebusch, K. Heyde, D. Van Neck, and M. Waroquier, Nucl. Phys. A503, 694 (1989).

[17] M. Waroquier, J. Sau, K. Heyde, P. Van Isacker, and H.
Vinckx, Phys. Rev. C 19, 1983 (1979).

[18] M. Waroquier, K. Heyde, and G. Wenes, Nucl. Phys. A404, 269 (1989).

[19] M. Waroquier, J. Ryckebusch, J. Moreau, K. Heyde, N. Blasi, S.Y. van de Werf, and G. Wenes, Phys. Rep. 148, 249 (1987).

[20] G.F. Bertsch and S.F. Tsai, Phys. Rep., Phys. Lett. 18C, 125 (1975).

[21] S.F. Tsai, Phys. Rev. C 17, 1862 (1978).

[22] A.L. Fetter and J.D. Walecka, Quantum Theory of ManyParticle Systems (McGraw-Hill, New York, 1971).

[23] M. Hashimoto, Prog. Theor. Phys. 94, 663 (1995).

[24] M. Buballa, S. Droźdź, S. Krewald, and J. Speth, Ann. Phys. (N.Y.) 208, 364 (1991).

[25] G. Co' and S. Krewald, Phys. Lett. 137B, 145 (1984).

[26] K. Langanke, Nucl. Phys. A621, 351c (1997).

[27] H.-T. Janka and W. Hillebrandt, Astron. Astrophys., Suppl. Ser. 78, 373 (1989).

[28] H.-T. Janka and W. Hillebrandt, Astron. Astrophys. 224, 49 (1989).

[29] G. Co' and S. Krewald, Nucl. Phys. A433, 392 (1985).

[30] D.J. Rowe, Nuclear Collective Motion (Methuen, London, 1970). 